\title{
Communication publique territoriale et approche générationnelle. Analyse comparée entre la France et le Québec
}

Entretien réalisé par Agnès Pecolo

\section{Agnès Pecolo}

\section{OpenEdition}

Journals

Édition électronique

URL : http://journals.openedition.org/communicationorganisation/3617

DOI : 10.4000/communicationorganisation.3617

ISSN : 1775-3546

\section{Éditeur}

Presses universitaires de Bordeaux

\section{Édition imprimée}

Date de publication : 1 décembre 2011

Pagination : 185-190

ISBN : 978-2-86781-745-8

ISSN : 1168-5549

Référence électronique

Agnès Pecolo, «Communication publique territoriale et approche générationnelle. Analyse comparée entre la France et le Québec », Communication et organisation [En ligne], 40 | 2011, mis en ligne le 01 décembre 2014, consulté le 01 mai 2019. URL : http://journals.openedition.org/ communicationorganisation/3617 ; DOI : 10.4000/communicationorganisation.3617

Ce document a été généré automatiquement le 1 mai 2019.

(C) Presses universitaires de Bordeaux 


\title{
Communication publique territoriale et approche générationnelle. Analyse comparée entre la France et le Québec
}

Entretien réalisé par Agnès Pecolo

\author{
Agnès Pecolo
}

1 Didier Rigaud est spécialiste de la communication publique territoriale. Maître de conférences associé à l'ISIC (Institut des Sciences de l'Information et de la Communication de l'Université de Bordeaux 3), ilest consultant associé à l'agence Epiceum (agence spécialisée en communication publique). Il collabore régulièrement avec différents organismes privés et publics, (CNFPT, Cap'Com, Territorial...) mais aussi depuis plus de 20 ans avec l'ACMQ, l'Association des Communicateurs Municipaux du Québec, province canadienne où il a débuté sa carrière professionnelle.

Les collectivités territoriales sont de plus en plus tentées par la segmentation de leur population selon les critères âge et cycles de vie lorsqu'elles communiquent. Qu'en est-il au Québec?

Les compétences des municipalités québécoises (l'équivalent de nos communes) diffèrent quelque peu de celles des communes françaises ${ }^{1}$.

Les collectivités territoriales françaises disposent, contrairement à leurs homologues outre Atlantique, des compétences suivantes: la petite enfance, la gestion des bâtiments des établissements scolaires, les écoles élémentaires, collèges et lycées, les activités périscolaires ainsi que la gestion de l'action sociale.

De fait, les publics jeunes et seniors sont plus l'objet de communication spécifique de la part des collectivités locales en France qu'au Québec.

Il est très fréquent de voir des rubriques ou des articles relatifs à la petite enfance, les enfants, les jeunes et les seniors dans les supports d'informations des collectivités françaises (bulletins et magazines papier, sites Internet, plaquettes, dépliants, etc.). On 
constate d'ailleurs certains stéréotypes dans la presse territoriale, une majorité de photographies de personnes âgées sont présentes dans les pages liées à l'action sociale et la santé alors qu'une représentation des enfants et jeunes domine dans les pages loisirs, culture et sport...

Alors qu'au Québec les actions de communication en direction de ces publics générationnels sont le plus souvent ponctuelles et liées à des objectifs conatifs.

En France plusieurs collectivités ont mis en place des supports spécifiques d'information pour des publics jeunes ou seniors comme par exemple la ville de Montpellier ${ }^{2}$ qui réalise une publication trimestrielle et un site Internet spécifique, Tip Top, destinés aux jeunes habitants ainsi que Tempo, le trimestriel destiné aux seniors montpelliérains. Ce type de publications n'est pas l'apanage des grandes collectivités. Des communes plus modestes par la taille développent également des supports d'information spécifiques segmentés, citons par exemple la ville de Royan et son « mag » jeune Koi 29 Royan $^{3}$. Les municipalités québécoises ne publient pas des supports d'information généralistes ciblés sur une génération spécifique.

Vous évoquiez les photos publiées dans la presse territoriale française: I'utilisation des images est-elle moins stéréotypée dans les supports de communication publique québécois?

Cette utilisation de photographies d'enfants, de jeunes et de seniors est plus rare dans la presse municipale québécoise. Ce qui peut déjà s'expliquer par un format moins "magazine» tant dans sa forme que son contenu. Support d'information administrative plus que de communication, une logique de service pratique domine. Mais c'est aussi dû, comme nous l'avons dit précédemment, du fait d'un champ de compétences moins en relation directe avec une segmentation générationnelle.

Néanmoins, force est de constater que les photos représentant par exemple les seniors dans les outils de communication publique des municipalités québécoises sont quasi identiques à celles que l'on trouve dans la presse territoriale française, voire parfois identiques quand elles sont issues de banque d'images. Ils sont représentés comme souriants, actifs, dynamiques, jamais seuls, en couple ou en groupe.

L'illustration utilisée pour la page internet de la rubrique "la Place des aînés » de la ville de Laval est tout à fait représentative. Elle montre un couple de personnes âgées devant le bâtiment du centre socioculturel, en jeans, tous deux sur un scooter vert anis. Il s'agit d'une photo montage car ce même couple en scooter a été vu dans une publicité pour une mutuelle de santé dans la presse française.

\section{Et côté jeunesse?}

Là aussi, peu d'illustrations avec des petits-enfants et enfants dans les supports d'information des municipalités québécoises mis à part dans les photos de familles ou en accompagnement des agendas culturels et activités de loisirs, certaines visant ces publics spécifiques (citons entre autres la fête de la lecture et du livre jeunesse de la ville de Longueuil $\left.{ }^{4}\right)$.

Par ailleurs, comme pour les seniors, les représentations des jeunes sont systématiquement positives. Ils sont actifs, créatifs, forces de projets, représentés en groupe, souriants... De nombreuses images proviennent également de banques d'images extérieures. Dans le domaine de la prévention en revanche, peu d'images sont utilisées 
et lorsqu'elles le sont, comme pour le site de prévention des gangs de rue ${ }^{5}$, on cherche clairement à éviter, au-delà du souci du droit à l'image, la stigmatisation d'une « tribu » de jeunes (une seule photo, intégrée au logo « choisis ton gang » et représentant trois jeunes vus de dos).

Vous avez parlé d'une place des "aînés » : use-t-on des mêmes dénominations quand il s'agit de parler de et au « petit » ou « vieux » public ?

L'étude comparative de sites Internet de municipalités québécoises et des communes françaises permet de constater quelques différences d'ordre sémantique. On retrouve très fréquemment sur les sites français des onglets ou espaces intitulés jeunes (ou jeunesse) et seniors.

Sur les sites des municipalités québécoises ces termes n'apparaissent pas. Il ne semble pas y avoir de cloisonnement par l'âge mais une seule entrée, la famille, le terme usité étant alors : «espace famille ». Dans quelques sites on peut voir apparaître le vocable « aînés » et non senior ou personnes âgées plus fréquents en France. La ville de Laval', seconde commune en nombre d'habitants du Québec, dispose d'un centre socioculturel et sportif pour les plus de 50 ans dont le nom est en effet « Place des aînés ».

Les personnes entre 60-65 ans notamment, rechignent souvent à être nommées «seniors». Elles ne se reconnaissent pas dans ce qualificatif et refusent d'être différenciées. Rencontre-t-on les mêmes réticences dans la population québécoise?

Je ne dispose pas d'éléments me permettant de répondre à cette question; je ne sais pas s'il en est de même avec le mot aîné, même si je pense que oui. Un rapport du gouvernement fédéral sur les aînés fait état de la difficulté de définir objectivement les termes "vieux ", " aîné » et " personne âgée " 7 . Il retient néanmoins le seuil de 65 ans arguant du fait que la plupart des gens ainsi que les institutions sociales admettent que c'est l'âge, reconnu comme âge «normal » de la retraite, auquel une personne devient un aîné. À 65 ans, une personne a le droit de recevoir une pension complète au Canada, même si bien des gens prennent leur retraite ou reçoivent leur pleine pension avant cet âge.

$\mathrm{Au}$ cours des années à venir, tout spécialement lorsque les premiers baby-boomers auront 65 ans, il se peut qu'une nouvelle définition du terme " aîné » remplace celle qui prévaut actuellement. Néanmoins et d'ici là, le terme ainé renvoie à toutes les personnes âgées de 65 ans et plus.

On trouve en France une distinction assez claire entre le troisième et le quatrième âge, les seniors et la grande vieillesse, les premiers faisant souvent l'objet d'une politique de loisirs "actifs» quand les seconds sont plus associés aux questions de santé et d'accompagnement. Retrouve-t-on cette coupure au Québec?

Dans les contenus de communication publique territoriale ces distinctions ne sont pas apparentes; comme nous l'avons dit précédemment les publics seniors sont unifiés en un seul vocable, "les seniors" en France, "les aînés" au Québec. Même si effectivement certains messages, informations sur les loisirs, s'adressent plus particulièrement aux jeunes retraités et les messages de prévention au quatrième âge, la sémantique qui accompagne ces communications ne fait pas ressortir de segmentation. 
Peut-on, malgré, on l'a compris, des compétences des municipalités moins en prise directe avec les cycles de vie, repérer une véritable politique de communication spécifique en fonction des âges de la vie?

Oui, même si l'initiative est gouvernementale. Les municipalités, le niveau administratif le plus proche des habitants, relaient et portent ces politiques segmentées au niveau provincial. La démarche MADA, Municipalité Amie des Aînés, en est un exemple. La municipalité de Laval communique sur cette démarche sur son site qui explique en quoi, initiée par le ministère de la Famille et des Aînés, elle vise à reconnaître une municipalité démontrant son souci des aînés en adaptant ses politiques, ses programmes, ses services et ses structures aux besoins de ceux-ci. Les municipalités amies des aînés agissent également en habitation, en loisirs, en aménagement urbain, en transport et en sécurité, et travaillent à mettre un frein à l'âgisme, un obstacle important au vieillissement actif. La ville en est actuellement dans une première phase de diagnostic lui permettant par la suite d'établir un plan d'actions en matière de politique en direction des aînés. Elle organise chaque année une semaine Lavalloise des aînés, évènement offrant diverses activités aux aînés afin de souligner leur rôle et leur apport dans la vie de la municipalité.

Mais le plus souvent, la communication en direction des seniors se limite à de l'information sur les activités de loisirs et services mis en place pour ces publics.

Montée en puissance de la prise en compte des «aînés » donc, comme en France où l'on met régulièrement en avant l'impossibilité d'ignorer aujourd'hui cette population qui compte démographiquement, et sujet de nombreux projets. Et la jeunesse, traditionnellement «ciblée » par les collectivités françaises depuis des années?

La jeunesse (adolescence et jeunes adultes) est présente dans la communication publique des municipalités québécoises. Elle fait l'objet comme en France de campagne de prévention et de sensibilisation (santé et sécurité), bénéficie d'offres de loisirs, d'animation. On peut y repérer quelques spécificités comme le phénomène des gangs de rue évoqué plus haut, réalité montréalaise. Le ministère de la Sécurité publique a mis en place toute une campagne de communication, portail Internet, promotion d'initiatives de sensibilisation, relayées par plusieurs municipalités de la métropole montréalaise. Autre exemple de politique de prévention, le centre de communication en santé mentale d'un centre hospitalier de Montréal, en partenariat avec la direction de la santé publique de la ville, a réalisé une trousse d'intervention sur les relations amoureuses des jeunes intitulée «Premiers Amours ». Cet outil est composé de cahiers d'animation, CD-ROM et DVD à utiliser par des professionnels de la prévention et de l'animation jeunesse.

L'âgisme, cité précédemment, est-il une discrimination à laquelle les collectivités sont particulièrement attentives?

Dans leur politique très certainement mais dans leur communication il en est tout autre.

La presse territoriale est souvent surnommée "la presse du bonheur »; elle demeure avant tout un outil de communication dont l'objectif est de valoriser le territoire et le projet politique pour ce même territoire. Que ce soit au Québec ou en France, les politiques, les actions mises en place en direction de la grande vieillesse y sont rarement traitées mis à part certains messages de prévention, par exemple sur la canicule, ces messages s'adressant plutôt aux proches et sont souvent factuels sous la forme de brèves. Tout comme la fin de vie, sujet très rarement abordé dans la 
communication publique territoriale et quand il l'est, c'est plutôt sous un angle « technique ", démarches, gestion des cimetières, etc.

Vous avez beaucoup évoqué la famille, elle semble être un axe de communication favorisé au Québec?

Effectivement, très souvent les politiques en direction des enfants sont diluées dans une politique plus globale en direction des familles, comme à la ville de Québec qui édite une plaquette intitulée " politique familiale ».

Il est intéressant de constater que cette ville met en avant le label «municipalité amie des enfants ", concept importé au Québec par le $\mathrm{CAMF}^{8}$. Sur la page d'accueil du site Internet du CAMF, l'image en bandeau présente trois générations d'une même famille, enfants, parents et grands-parents mais sur les quatre grands titres de rubriques seuls les aînés apparaissent, l'enfance et la jeunesse étant intégrées dans la «famille ».

L'entrée par le vocable famille donne une impression de continuité de la vie et non de juxtaposition de classes d'âges, à l'image du gouvernement du Québec qui pour accompagner sa «Stratégie d'action jeunesse 2009-2014» utilise l'accroche ou slogan : «Enrichir le Québec de sa relève ».

Le Québec fait le choix finalement d'un positionnement plus intergénérationnel?

Oui, en effet il y a cette "entrée " par la famille ; les municipalités appréhendent les services rendus et communiquent en direction de la famille, tous âges confondus comme un continuum de service public et d'accompagnement tout au long de la vie. Alors qu'en France on a plus souvent une segmentation par génération, celle-ci se traduisant même comme nous l'avons dit auparavant par une multiplication des supports d'information.

L'intergénérationnel en France illustre plus souvent des actions liées au développement durable ou actions d'Agenda 21.

À l'instar de la communication publique française, les illustrations accompagnant les supports intergénérationnels véhiculent l'idée de transmission de connaissances ou de savoir-faire entre générations, comme cette photo représentant un grand-père et sa petite fille face à un écran d'ordinateur, illustrant un article du site Internet du gouvernement du Québec sur les cinq générations d'internautes9.

La thématique du « vivre ensemble » et de la cohésion sociale est très prégnante dans la communication territoriale en France. Qu'en est-il au Québec?

En France cette thématique du vivre ensemble est plus prégnante depuis que les collectivités appuient la dimension sociale des politiques de développement durable. Au Québec le développement durable fait moins l'objet de politiques affirmées, le vivre ensemble apparaît sous l'angle de la famille et de son épanouissement sur le territoire grâce aux projets mis en place par les élus. 


\section{NOTES}

1. Elles sont les suivantes : aménagement du territoire et urbanisme/parcs et espaces naturels/ loisirs et culture/matières résiduelles (gestion des déchets)/eau potable et assainissements des eaux/services policiers/pompiers/réseau routier/transports en commun/ habitation (logement).

2. Consulter les sites Internet de la ville de Montpellier : http://tiptop.montpellier.fr et http:// www.montpellier.fr

3. Revue Koi29 Royan, site de la revue Koi29Royan, [En ligne], cf. http://www.ville-royan.fr/? page=jeunesse/koi29royan.

4. Consulter le site de la ville de Longueil : http://www.longueuil.ca.

5. Consulter le site de prévention des gangs de rue: http://www.gangsderue.gouv.qc.ca/plangouvernemental/axe2-preventionintervention.html.

6. Consulter le site du centre socioculturel et sportif pour les plus de 50 ans de la ville de Laval : http://www.placedesaines.org/.

7. Un portrait des aînés au Canada, rapport de Statistique Canada, n 89-519-XIF.

8. Carrefour Action Municipale et Famille, en charge de la promotion, de la mise en œuvre, de l'accompagnement et du suivi des politiques familiales municipales. Cet organisme à but non lucratif est également à l'initiative du MADA évoqué précédemment.

9. Les 18-34 ans (génération Y) sont considérés comme des utilisateurs intensifs d'Internet ; les 35-44 ans (génération X) comme des enthousiastes pratiques; les 45-54 ans (jeunes boomers) comme les reflets de la tendance centrale; les 55-64 ans (les boomers) comme des utilisateurs prudents et les 65 ans et plus (les aînés) comme des néophytes réservés ou « seniornautes ». 